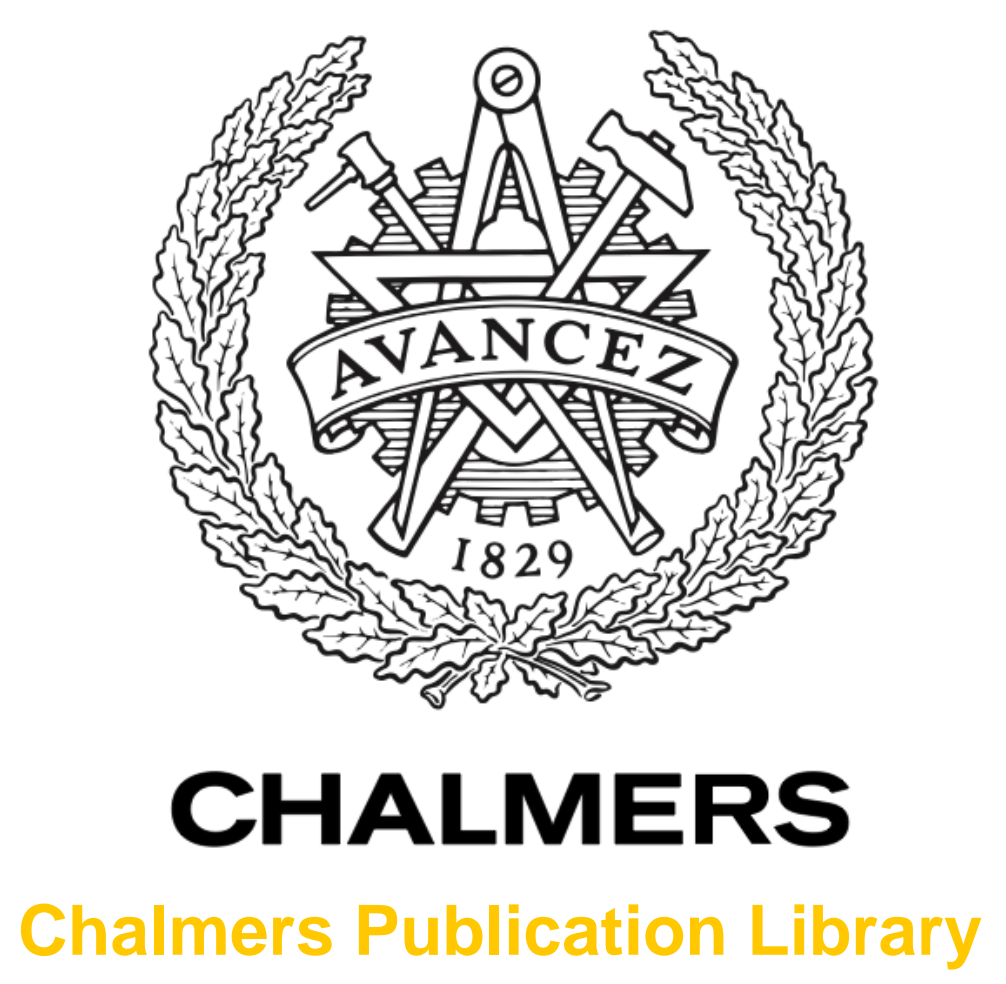

Sub-sampled OFDM based sub-band ultra-wideband system

This document has been downloaded from Chalmers Publication Library $(\mathrm{CPL})$. It is the author's version of a work that was accepted for publication in:

IEEE Wireless Communications and Networking Conference, WCNC 2012, Paris, 1-4 April 2012 (ISSN: 1525-3511)

Citation for the published paper:

Nair, J. ; Sen, D. ; Jos, S. (2012) "Sub-sampled OFDM based sub-band ultra-wideband system". IEEE Wireless Communications and Networking Conference, WCNC 2012, Paris, 1-4 April 2012 pp. 48-53.

http://dx.doi.org/10.1109/WCNC.2012.6214416

Downloaded from: http://publications.lib.chalmers.se/publication/163184

Notice: Changes introduced as a result of publishing processes such as copy-editing and formatting may not be reflected in this document. For a definitive version of this work, please refer to the published source. Please note that access to the published version might require a subscription.

Chalmers Publication Library (CPL) offers the possibility of retrieving research publications produced at Chalmers University of Technology. It covers all types of publications: articles, dissertations, licentiate theses, masters theses, conference papers, reports etc. Since 2006 it is the official tool for Chalmers official publication statistics. To ensure that Chalmers research results are disseminated as widely as possible, an Open Access Policy has been adopted.

The CPL service is administrated and maintained by Chalmers Library. 


\title{
Sub-sampled OFDM based Sub-band Ultra-Wideband System
}

\author{
Jinesh P Nair, Debarati Sen*, Sujit Jos and Arun Naniyat \\ SAIT India Labs, Advanced Technology Team, Samsung Electronics, India \\ Signal and Systems Lab, Chalmers University of Technology, Sweden* \\ \{jinesh.p, sujit.jos, arun.naniyat\}@ samsung.com, debarati@chalmers.se*
}

\begin{abstract}
In sub-band ultra-wideband (SUWB) systems, the use of spreading codes in conjunction with sub-banding enables energy efficient reduced sampling rate receiver designs. In this work, the orthogonal frequency division multiplexing (OFDM) technique is proposed for SUWB systems as a means to mitigate the multipath fading effects of the channel. The OFDM demodulation performed at the sub-sampled rate with reduced number of discrete Fourier transform (DFT) points provides scope for low power receiver implementations. Moreover, OFDM improves the flexibility as bandwidth resources can be allocated with improved granularity at integral multiples of the OFDM sub-channel bandwidth. The requisite correlation properties of the spreading codes is relaxed in the proposed OFDM-SUWB system and more number of spreading codes can be used when compared to the existing SUWB system. Also, a simple channel estimation method exploiting the low complexity advantage of the inherent spreading code based receiver is proposed. Simulation results in terms of the bit error rate (BER) performance are presented over the IEEE 802.15.4a channel models and also comparisons with the multi-band OFDM (MB-OFDM) system are made demonstrating the usefulness of the proposed scheme.
\end{abstract}

Index Terms-Sub-sampled OFDM, UWB, Sub-banding.

\section{INTRODUCTION}

Among the existing ultra wideband (UWB) systems, impulse radio UWB (IR-UWB) supports low data rates of the order of a few Mbps and multi-band orthogonal frequency division multiplexing (MB-OFDM) systems support very high data rates of the order of $500 \mathrm{Mbps}$ [1], [2]. In order to address the data rate requirements intermediate to these two, recently a Sub-band based Ultra-wideband System (SUWB) was proposed for energy efficient medium data rate communications over short ranges [3] and [4]. It was seen that significant power savings of the order of around $30 \%$ can be obtained in such systems in comparison to full band systems with scalable data rates ranging from $12.5 \mathrm{Mbps}$ to $62.5 \mathrm{Mbps}$.

Such systems are highly attractive in applications wherein scalability of services with energy efficiency is critical as in next generation connected healthcare and personal space communications [5]. The technique provides scope for using the UWB bandwidth efficiently by exploiting the available link margin for short range communications. Furthermore, the SUWB system facilitates energy efficient receiver designs through reduced sampling rate techniques.

However, the existing SUWB system in [3] and [4] do not incorporate methods to mitigate the multi-path effects induced by the UWB channel. The performance degradation is very severe when the data rate increases. There is a need to combat the channel multipath fading effects at the rate of the sub-band bandwidth (also called the sub-sampled rate) or below.

As a solution, we propose a sub-sampled OFDM based subband UWB (SUWB) system in this paper (OFDM-SUWB). Here OFDM is used on a per-sub-band basis for channel mitigation at the sub-sampled rate. The OFDM-SUWB system proposed here has several advantages compared to the existing SUWB system in [3] and [4]. The existing SUWB system calls for the codes to have ideal correlation properties at non-zero lags. The number of codes satisfying this property is far less compared to the spreading factor [9]. In OFDMSUWB, flat fading on each sub-carrier eases this requirement. Good correlation properties are required only at zero lag. It is easier to have more number of spreading codes satisfying this property and this further improves the scalability. Earlier, the granularity in resource allocation was limited by the sub-band bandwidth. Now, resources can be allocated at integral multiples of the OFDM sub-channel bandwidth thus significantly improving the flexibility. Moreover, the proposed OFDM demodulation handles the multipath fading channel with a fewer number of discrete Fourier transform (DFT) points as it is done on the sub-band bandwidth rather than the full UWB bandwidth at the receiver. The channel estimation and one tap equalization can be carried out after the despreading process. This is advantageous as the effect of the fading is removed on the requisite sub-bands rather than on the entire full band.

Simulation results in terms of the bit error rate (BER) performance demonstrating the usefulness of the proposed scheme are presented over the IEEE 802.15.4a channel models. Moreover, comparisons with MB-OFDM over the IEEE 802.15.3a channels indicate that a similar performance can be achieved with the additional power saving advantage for medium data rates of around 54 Mbps.

The rest of the paper is organized as follows. Sec. II introduces the OFDM-SUWB system. Sec. III describes the OFDM-SUWB transmitter and receiver. Sec. IV presents the simulation results and conclusions are drawn in Sec. V.

\section{Sub-SAMPLED OFDM BASED SUWB SySTEM}

\section{A. Background}

In the existing UWB systems, the transmitted power is distributed across the whole $500 \mathrm{MHz}$ band uniformly. This is an inefficient way of utilizing the UWB bandwidth especially 
when the link margin is high. Some of the bandwidth may be released for other purposes by reducing the link margin. The link margin in this scenario is the difference between the maximum transmit power possible in the whole band chosen following FCC directives and the minimum transmit power.

The concept of sub-banding proposed in [3], [4] for UWB systems facilitates this control of bandwidth and transmit power. Here, the UWB bandwidth $B \geq 500 \mathrm{MHz}$ is divided into $N$ number of sub-bands. With the efficient use of these sub-bands the SUWB system is able to achieve power efficient communication with medium data rates over short ranges. Flexibility is incorporated into the SUWB system as the subbands can be used to increase data rate or improve the BER performance by exploiting the frequency diversity or provide multi-user support. Furthermore, the SUWB system facilitates low power implementations by reducing the sampling rate requirements by $B / N \mathrm{~Hz}$ at the receiver. This is by using an orthogonal spreading code based interference rejection and multi-path cancellation receiver. This obviates the need for individual down-conversion and filtering of the sub-bands.

\section{B. Motivation for an OFDM based SUWB System}

The performance of the SUWB system degrades in the presence of multi-path fading UWB channels as the data rate requirement increases. There is a need to mitigate the channel impairments at the rate of the sub-band bandwidth without significantly increasing the complexity. Multiple options can be envisaged. One is that of using a full band equalizer. This would significantly reduce the advantage of the existing system which is that of reduced sampling rate receiver designs.

The other is to estimate the full band equalizer coefficients, and perform the linear equalization in the analog portion [6]. Although better than a full band equalizer implementation, this has the disadvantage of having separate data and preamble analog to digital converters (ADCs). This increases the cost in terms of power and area and also there is a problem of fast switching between these two ADCs. Another option is to formulate a multi-channel block equalization structure to invert the composite sub-sampled channel at the rate of the sub-band bandwidth. However, such an equalizer is similar in complexity to the full band equalizer and has increased latency.

These disadvantages motivates the need for a solution at the sub-sampled rate i.e. $(B / N)$. The proposed sub-sampled OFDM based SUWB system (OFDM-SUWB) described next is an elegant solution with the additional benefits of enhanced scalability in resource allocation.

\section{Brief System Description}

In OFDM-SUWB, the UWB bandwidth of $B \geq 500 \mathrm{MHz}$ is divided into $N$ fixed number of sub-bands. The OFDM modulation for each of these sub-bands divides the sub-bands into a number of sub-channels. The modulated data symbols are spread using orthogonal codes to occupy the bandwidth of the OFDM sub-channels. The signal consisting of a plurality of such sub-bands is transmitted through a UWB channel. At the receiver, the RF section receives the UWB signal but the ADC samples this signal at the rate of the sub-band bandwidth.
The resulting signal is a composite signal band-limited to the sub-band bandwidth along with the aliases. An OFDM demodulation is performed on the composite signal and a subcarrier wise despreading operation is performed. The unwanted symbols are removed due to the orthogonality of the codes. The desired signals are then recovered using the demodulation and detection processes.
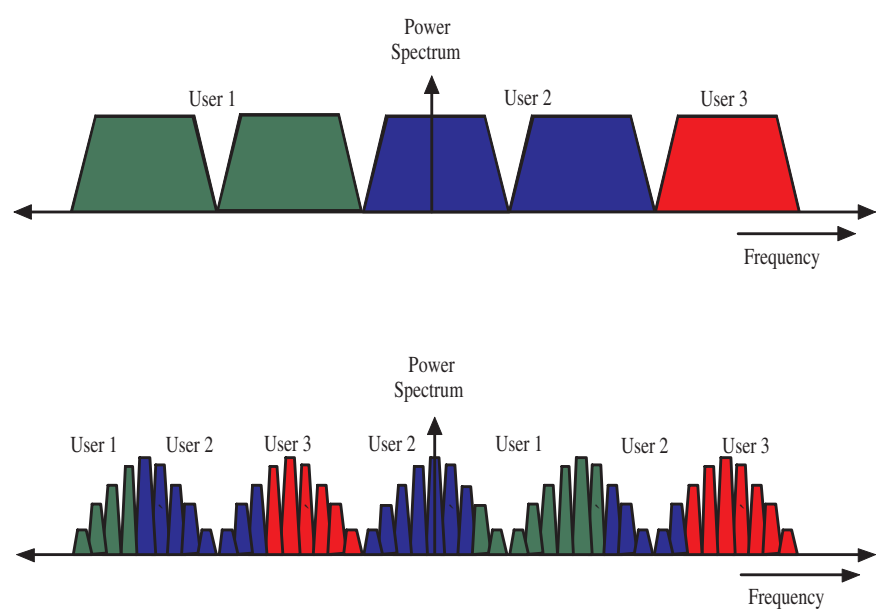

Fig. 1. Illustration of the key differences between SUWB and OFDM-SUWB The first figure shows user allocation in SUWB. Codes allocated on per subband basis. Smallest resource unit is a sub-band. The second figure shows user allocation in OFDM-SUWB. Codes allocated on per sub-carrier basis. Smallest resource unit is a sub-carrier.

\section{OFDM-SUWB: DETAILED DESCRIPTION}

The proposed OFDM-SUWB system model is described here including the details of the transmitter and receiver.

\section{A. OFDM-SUWB Transmitter}

The UWB bandwidth $B$ is divided into $N$ sub-bands of bandwidth $B / N=1 / T_{0}$. Here $T_{0}$ is the time resolution of the sub-band signals. An OFDM operation is performed on each of the sub-bands. The bandwidth of each of the subcarrier signals is $B /\left(N \times N_{b}\right)$ where $N_{b}$ is the number of sub-carriers. The sub-carrier signals are obtained by spreading the data signals with the spreading codes associated with each sub-band.

Let $S_{k}^{i}\left(n T_{c}\right)$ be the modulated symbols formed from the incoming binary data for the $k^{t h}$ sub-band and the $i^{\text {th }}$ subcarrier having symbol duration $T_{c}$. These are then multiplied by the orthogonal spreading code $C_{k}^{i}\left(n T_{b}\right)$ associated with the $k^{\text {th }}$ sub-band and the $i^{\text {th }}$ sub-carrier having chip duration $T_{b}=N \times N_{b} / B=N_{b} T_{0}$ and spreading factor $P=T_{c} / T_{b}$. If each OFDM symbol is multiplied with the same chip of the spreading code associated with the $k^{\text {th }}$ sub-band then

$$
\begin{aligned}
C_{k}^{i}\left(n T_{b}\right) & =\lambda_{k} \quad \forall i \in\left[0, N_{b}-1\right] \\
& =C_{k}^{i}\left[(n+P) T_{b}\right]
\end{aligned}
$$

$\lambda_{k}$ is one of the elements of the chip sequence. Using OFDM along with sub-banding allows allocation of codes on a per sub-carrier basis. Use of OFDM also allows flexibility in 
bandwidth allocation. The bandwidth allocated to a user with flexibility for diversity or rate enhancement may be formed by aggregating disjoint portions of the $500 \mathrm{MHz}$ UWB spectrum, even though the aggregated bandwidth is not an integer multiple of the sub-band bandwidth. This is unlike the system described in [3] and [4]. Moreover, the bandwidth resources can be allocated at integral multiples of the OFDM subchannel bandwidth. This improves the flexibility as resources can be allocated with finer granularity. This is in contrast with the system in described in [3] and [4], in which case the smallest unit for resource allocation is the sub-band bandwidth which is very large compared to the OFDM sub-channel bandwidth (Refer Fig. 1).

An inverse discrete Fourier transform (IDFT) operation is carried out for each sub-band signal using $N_{b}$ sub-carriers. This is expressed as

$$
\tilde{x}_{k}\left(n T_{0}\right)=\frac{1}{\sqrt{N_{b}}} \sum_{m=0}^{N_{b}-1} S_{k}\left(m T_{0}\right) C_{k}\left(m T_{0}\right) e^{j \frac{2 \pi m n}{N_{b}}}
$$

$S_{k}\left(m T_{0}\right)$ and $C_{k}\left(m T_{0}\right)$ can be related to $S_{k}^{i}\left(m T_{b}\right)$ and $C_{k}^{i}\left(m T_{b}\right)$ respectively in a number of ways depending on how the chips are allocated to the sub-carriers, either serially or with interleaving within a sub-band. A serial representation is as follows

$$
\begin{aligned}
& S_{k}^{i}\left(n T_{b}\right)=S_{k}\left[\left(i+n N_{b}\right) T_{0}\right], \quad i \in\left[0, N_{b}-1\right] \\
& C_{k}^{i}\left(n T_{b}\right)=C_{k}\left[\left(i+n N_{b}\right) T_{0}\right]
\end{aligned}
$$

A cyclic prefix (CP) is appended to the IDFT output to form the OFDM symbol. Alternatively for improved spectral characteristics, a zero padding (ZP) operation may be carried out [2]. The length of the cyclic prefix is decided based on the channel characteristics encountered. $\tilde{x}_{k}\left(n T_{0}\right)$ in (2) is then interpolated by a factor of $N$ to obtain $x_{k}\left(n T_{s}\right)$, where $T_{s}=1 / B$ are the samples of the UWB signal. Ideal low pass interpolation is assumed here. The sub-band signals are then combined to form the SUWB signal at base-band.

$$
z\left(n T_{s}\right)=\sum_{k=-\lfloor N / 2\rfloor}^{\lfloor N / 2\rfloor} x_{k}\left(n T_{s}\right) e^{j 2 \pi f_{0} k n T_{s}}
$$

$z\left(n T_{s}\right)$ spans the UWB bandwidth of $500 \mathrm{MHz}$ or more. The design choice of the OFDM parameters can be done on the basis of the sub-band bandwidth and associated sampling rates [10]. The signal in (3) is similar to the MB-OFDM waveform for one of the multi-bands. In the special case, when the spreading codes are absent, the signal in (3) is the same as the MB-OFDM system for a single UWB band [2]. However, the use of spreading codes along each sub-carrier enables power efficient reduced sampling rate receiver implementation as described next.

\section{B. Channel}

The transmitted baseband signal $z\left(n T_{s}\right)$ convolves with the channel impulse response $h\left(n T_{s}\right)$. We consider the UWB channels specified in IEEE 802.15.4a and IEEE 802.15.3a channel models [7] [8]. $h\left(n T_{s}\right)$ is expressed as

$$
h\left(n T_{s}\right)=\sum_{l=0}^{L} \sum_{v=0}^{V} a_{v, l} e^{j \phi_{v, l}} \delta\left(n T_{s}-T_{l}-\tau_{v, l}\right)
$$

Where $a_{v, l}$ is the tap weight of the $v^{t h}$ component in the $l^{t h}$ cluster, $T_{l}$ is the delay of the cluster and $\tau_{v, l}$ is the delay of the $v^{t h}$ multi-path component relative to the $l^{t h}$ cluster arrival time and the phases $\phi_{v, l}$ are uniformly distributed in the range $[0,2 \pi]$.

\section{OFDM-SUWB Receiver}

The received signal after being subjected to the additional additive white Gaussian Noise (AWGN) $w\left(n T_{s}\right)$ at the receiver front end is given by

$$
y\left(n T_{s}\right)=z\left(n T_{s}\right) * h\left(n T_{s}\right)+w\left(n T_{s}\right)
$$

The response $y\left(n T_{s}\right)$ can be equivalently represented as the sum of the individual sub-band responses.

$$
\begin{aligned}
y\left(n T_{s}\right)= & \left(\sum_{k=-\lfloor N / 2\rfloor}^{\lfloor N / 2\rfloor} x_{k}\left(n T_{s}\right) e^{j 2 \pi f_{0} k n T_{s}} *\right. \\
& \left.\sum_{k=-\lfloor N / 2\rfloor}^{\lfloor N / 2\rfloor} h_{k}\left(n T_{s}\right) e^{j 2 \pi f_{0} k n T_{s}}\right)+w\left(n T_{s}\right)
\end{aligned}
$$

Here $h_{k}\left(n T_{s}\right)$ represents the equivalent sub-band responses centered on zero frequency of the $k^{t h}$ sub-band. Since the individual sub-bands have very low out of band interference, the cross terms involved in the above convolution can be assumed to be negligible. Hence we can write the above as

$$
y\left(n T_{s}\right)=\sum_{k=-\lfloor N / 2\rfloor}^{\lfloor N / 2\rfloor} \sum_{m=0}^{Q-1}\left(x_{k}\left(n T_{s}-m T_{s}\right) e^{j 2 \pi f_{0} k n T_{s}} \times\right.
$$

Or equivalently

$y\left(n T_{s}\right)=\sum_{k=-\lfloor N / 2\rfloor}^{\lfloor N / 2\rfloor} x_{k}\left(n T_{s}\right) * h_{k}\left(n T_{s}\right) e^{j 2 \pi f_{0} k n T_{s}}+w\left(n T_{s}\right)$

The received signal is down sampled by a factor of $N$ to obtain samples spaced with separation $T_{0}$.

$$
\tilde{y}\left(n T_{0}\right)=y\left(n N T_{s}\right)
$$

This is the signal band-limited to $B / N$ and centred on zero and contains aliased sub-bands. ADCs that allow such a subsampling operation come under the class of under-sampled ADCs. Sub-sampling as in (9) at the rate of the sub-band bandwidth significantly reduces the power consumption due to the reduced sampling rate [4], [11]. However, the challenge is to discriminate the desired data symbols from the composite signal. The following discussion reveals an approach to do this on a per-subcarrier basis using spreading codes.

Under perfect synchronization, all the CP/ZP signals of the individual sub-bands align and can be commonly processed. After performing the appropriate processing for the CP/ZP portion of the composite signal a DFT operation is carried out at the receiver to obtain $Y\left(i T_{0}\right)$ on the $i^{t h}$ sub-carrier.

$$
Y\left(i T_{0}\right)=\frac{1}{\sqrt{N_{b}}} \sum_{n=0}^{N_{b}-1} \tilde{y}\left(n T_{0}\right) e^{-j \frac{2 \pi n i}{N_{b}}}
$$


From (10), we see that the DFT is performed after subsampling. Only a single DFT operation with reduced number of points $N_{b}$ at the reduced sampling rate of $B / N$ is required. A full band DFT system like MB-OFDM would require an $N \times N_{b}$ point DFT at the higher sampling rate of $B$ significantly increasing the power consumption. Substituting (8) and (9) in (10) we have

$$
\begin{gathered}
Y\left(i T_{0}\right)= \\
\frac{1}{\sqrt{N_{b}}} \sum_{n=0}^{N_{b}-1}\left[\sum_{k=-\lfloor N / 2\rfloor}^{\lfloor N / 2\rfloor} x_{k}\left(n T_{0}\right) * h_{k}\left(n T_{0}\right) e^{j 2 \pi f_{0} k n T_{0}}\right. \\
\left.\quad+w\left(n T_{0}\right)\right] e^{-j \frac{2 \pi n i}{N_{b}}}
\end{gathered}
$$

The above equation reveals the composite nature of the subsampled signal. Since a convolution in time is a multiplication in frequency domain, $Y^{i}\left(n T_{b}\right)$ i.e. the received symbols on the $i^{\text {th }}$ sub-carrier at the rate of the OFDM symbol duration can be expressed as

$$
Y^{i}\left(n T_{b}\right)=\sum_{k=-\lfloor N / 2\rfloor}^{\lfloor N / 2\rfloor} S_{k}^{i}\left(n T_{b}\right) C_{k}^{i}\left(n T_{b}\right) H_{k}^{i}\left(n T_{b}\right)+W^{\prime}\left(n T_{b}\right)
$$

Here $H_{k}^{i}\left(n T_{b}\right)$ is the channel gain for the $k^{t h}$ sub-band and the $i^{t h}$ sub-carrier. The relation between $Y^{i}\left(n T_{b}\right)$ and $Y\left(n T_{0}\right)$ when the data is serially allocated along sub-carriers as in Sec. III-A is given by

$$
Y^{i}\left(n T_{b}\right)=Y\left(i T_{0}+n N_{b} T_{0}\right)
$$

Interleaved sub-carrier allocations at the transmitter will have corresponding de-interleaving done at the receiver. Let the data that is to be decoded be spread with the code $C_{k}^{i}\left(n T_{b}\right)=$ $C_{0}^{i}\left(n T_{b}\right)$. The despreading (integration and dump) consists of the following operation

$$
\tilde{S}^{i}\left(n P T_{b}\right)=\frac{1}{P} \sum_{k=n}^{n+P-1} \tilde{Y}^{i}\left(k T_{b}\right) C_{0}^{i}\left(k T_{b}\right)
$$

The good correlation properties of the spreading codes ensure that the signals from the unwanted sub-bands are cancelled on each sub-carrier. That is

$$
\sum_{n=m}^{m+P-1} C_{k}^{i}\left(m T_{b}\right) C_{0}^{i}\left(m T_{b}\right)= \begin{cases}1, & k=0 \\ 0, & \forall k \neq 0\end{cases}
$$

From (12), it can be readily seen that the channel is flat fading on each sub-carrier. Good correlation properties are required only at zero lag unlike the earlier SUWB system which require such good properties for lags beyond zero. This enables the synthesis of more number of codes [9] when compared with the earlier SUWB system in [3], [4]. These large sets of codes can greatly enhance the flexibility of the system and paves the way for further enhancements like overloading to increase the data rate. From (12), (14) and (15), the resulting signal is now given by

$$
\tilde{S}^{i}\left(n P T_{b}\right)=H_{0}^{i}\left(n P T_{b}\right) S_{0}^{i}\left(n P T_{b}\right)+W^{\prime}\left(n P T_{b}\right)
$$

From (14) and (16), it is seen that the wanted signals on each sub-carrier can be easily discriminated without the need for individual down-conversion and filtering of the sub-bands. Also, the above equations reveal an advantage vis-a-vis the channel estimation problem. It can be seen that all the subband channels on each sub-carrier need not be estimated. Only the channel corresponding to the desired sub-band needs to be estimated on each sub-carrier. Moreover this needs to be done after despreading and hence at a further reduced rate of $B /\left(N N_{b} P\right)$. Hence the channel estimation and compensation scheme is greatly simplified. After the channel $\hat{H}_{0}^{i}\left(n P T_{b}\right)$ is estimated, a zero forcing (ZF) equalizer is applied as

$$
\hat{S}^{i}\left(n P T_{b}\right)=\frac{\tilde{S}^{i}\left(n P T_{b}\right)}{\hat{H}_{0}^{i}\left(n P T_{b}\right)}
$$

Here $\hat{S}^{i}\left(n P T_{b}\right)$ are the equalized symbols. These are then passed through the typical stages of a communication receiver.

\section{Channel Estimation Method}

The channel estimate is carried out at the modulated symbol rate after despreading. In the transmitter, in the preamble portion, for the channel estimation slot, one white training sequence is generated in the frequency domain. This when spread with the spreading sequence associated with each subband along each sub-carrier, forms the channel estimation sequence for each sub-band.

Let a basic white training sequence out of which the sequences for each sub-band is generated be represented as $T^{i}\left(n T_{b}\right)$. The training sequence for each sub-band in the frequency domain is obtained by spreading the white training sequence with the corresponding chip sequence associated with a sub-band along each sub-carrier. The channel estimation training sequence $T_{k}^{i}\left(n T_{b}\right)$ is given by

$$
T_{k}^{i}\left(n T_{b}\right)=T^{i}\left(n T_{b}\right) C_{k}^{i}\left(n T_{b}\right)
$$

$T^{i}\left(n T_{b}\right)$ here can be used to improve the peak to average power ratio (PAPR) characteristics of the training sequence. For example, in the event the allocation of spreading codes is as in (1), the PAPR of the time domain sequence is very high. $T^{i}\left(n T_{b}\right)$ may be used in such cases for appropriately shaping the training sequence.

At the receiver, after proper acquisition of the training sequence preamble portion of the received signals, a despreading operation is carried out as in (14). A frequency domain least squares (LS) based channel estimation for the $i^{\text {th }}$ sub-carrier is given by

$$
\hat{H}_{0}^{i}\left(n P T_{b}\right)=\frac{\frac{1}{P} \sum_{k^{\prime}=n}^{n+P-1} \tilde{Y}^{i}\left(k^{\prime} T_{b}\right) T_{0}^{i}\left(k^{\prime} T_{b}\right)}{\left[T^{i}\left(n P T_{b}\right)\right]^{2}}
$$

Expanding the above equation we have

$$
\begin{aligned}
& \hat{H}_{0}^{i}\left(n P T_{b}\right)= \\
& \frac{\frac{1}{P} \sum_{k=n}^{n+P-1}\left[\sum_{k=-B_{0}}^{B_{0}}\left\{H_{k}^{i}\left(k T_{b}\right) T_{k}^{i}\left(k T_{b}\right)+W^{\prime}{ }_{k}\left(k T_{b}\right)\right\}\right] T_{0}^{i}\left(k T_{b}\right)}{\left[T^{i}\left(n P T_{b}\right)\right]^{2}}
\end{aligned}
$$

From the correlation properties in (15) and from (19) we have

$$
\hat{H}_{0}^{i}\left(n P T_{b}\right)=H_{0}^{i}\left(n P T_{b}\right)+W^{\prime \prime}\left(n P T_{b}\right)
$$


Hence we obtain the least squares estimate of the channel on each sub-carrier. The processing gain in the channel estimate comes from the spreading factor. From (18), (20) and (21), it can be seen that the training sequences used and the de-spreading operation at the receiver prior to the channel estimation, eliminate the unwanted channels and retain only the channel whose estimate is desired. This is advantageous as the effect of the fading is removed only on the requisite sub-bands rather than on the entire full band.

Further accuracy of the estimate can be obtained by making use of the fact that the channel memory is lower than the number of sub-carriers and hence the noise component on each sub-carrier can be reduced by filtering. This is exploited by circularly convolving the channel estimates on each sub-carrier with a sinc function as follows

$$
\bar{H}_{0}^{i}\left(n P T_{b}\right)=\hat{H}_{0}^{i}\left(n P T_{b}\right) \otimes \frac{\sin \left(\frac{2 \pi Q i}{N_{b}}\right)}{\left(\frac{2 \pi Q i}{N_{b}}\right)}, i \in\left[0, N_{b}-1\right]
$$

Here $Q$ is the number of channel taps. This improves the estimation accuracy. If $\sigma_{T}^{2}$ is the power of the training sequence and $\sigma_{w}^{2}$ is the noise variance, then the mean square estimation error variance can be shown to be $\sigma_{w}^{2} /\left(P \sigma_{T}^{2}\right)$ and $\left(Q \sigma_{w}^{2}\right) /\left(P N_{b} \sigma_{T}^{2}\right)$ for the estimators (19) and (22) respectively [12]. Hence there is an improvement by a factor of $N_{b} / Q$. For $Q<<N_{b}$, this is significant which is generally the case in OFDM.

\section{Simulation Results}

The simulation results in terms of the BER vs. signal to noise ratio (SNR) in $\mathrm{dB}$ for the sub-sampled OFDM SUWB system are presented in this section. The simulation parameters for both the cases considered here are tabulated in Table I. From the table the remaining parameters can be easily

TABLE I

Simulation PARAMETERS

\begin{tabular}{|c||c||c|}
\hline Sr.No & Simulation Parameter & Value/Type \\
\hline \hline 1 & UWB Bandwidth $(B)$ & $500 \mathrm{MHz}$ \\
\hline 2 & Number of Sub-bands $(N)$ & 5 \\
\hline 3 & Number of FFT $\left(N_{b}\right)$ & 32 \\
\hline 4 & CP length & 3 Samples \\
\hline 5 & Spreading Code & $\begin{array}{c}\text { Walsh- } \\
\text { Hadamard }(\mathrm{WH})\end{array}$ \\
\hline 6 & Spreading Factor $(P)$ & 8 \\
\hline 7 & Modulation Type & BPSK, QPSK \\
\hline
\end{tabular}

deduced. The OFDM symbol duration without the CP is 320 ns. The duration of the CP is $30 \mathrm{~ns}$. The subcarrier bandwidth is $3.125 \mathrm{MHz}$ and the BPSK modulation on each subcarrier is carried out at the rate of $390.625 \mathrm{KHz}$. The overall data range support for this case varies from approximately $11.5 \mathrm{Mbps}$ $(500 \times 320 /[5 \times 350 \times 8])$ to $91.5 \mathrm{Mbps}(500 \times 320 /[5 \times 350])$. The lower range is set when the 5 sub-bands transmit the same data. The higher range is set when the 5 sub-bands transmit different data streams. Since it is more critical to study the performance of OFDM when the data rate is higher, we evaluate the performance when different sub-bands transmit different data and hence the frequency diversity order is the lowest i.e. 1. The channel estimation method described in Sec. III-D is employed. Perfect synchronization is assumed.

Since each sub-band performs a 32 point DFT at $100 \mathrm{MHz}$, composite UWB signal of $500 \mathrm{MHz}$ has an equivalent $(5 \times 32)$ point DFT. However at the receiver only a 32 point DFT is performed at $100 \mathrm{MHz}$ and the aliased unwanted sub-bands are rejected using the 8 length WH codes. Since the maximum rms delay spread considered here is $11 \mathrm{~ns}$, a CP of 3 samples is good enough for sufficient multipath energy collection [2].

Figure 2 shows the BER vs. SNR performance of the OFDM-SUWB based system. BPSK modulation is used. For a comparison, the performance of the single carrier (SC) SUWB system without equalization and also with a $500 \mathrm{MHz}$ linear equalizer (LE) is shown. The IEEE 802.15.4a channel model (CM) 3 with a delay spread of $11 \mathrm{~ns}$ is used to demonstrate the results [7]. The $500 \mathrm{MHz} \mathrm{LE}$ is fractionally spaced and is a zero forcing (ZF) solution with 35 taps. This is indeed highly complex and power consuming. As is evident the OFDM-SUWB outperforms the SC system for all SNR regions of interest. When compared to the SC with LE, there is a cross over point at $13 \mathrm{~dB}$. This is because of the loss in multipath diversity in uncoded OFDM transmission resulting in performance degradation especially at the channel nulls. In the SC system with fractionally spaced LE at $500 \mathrm{MHz}$, the time diversity is well exploited, thus explaining the improved performance in the high SNR regime albeit with increased complexity. For a BER of $10^{-2}$, the OFDM-SUWB system outperforms the SUWB SC-LE system by $2 \mathrm{~dB}$ and the SUWB system without equalization by $8 \mathrm{~dB}$.

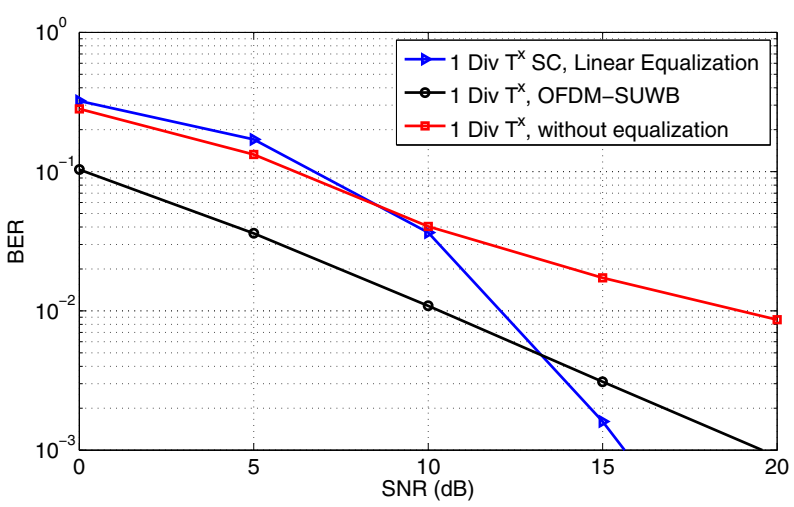

Fig. 2. BER vs. SNR performance comparison of the OFDM SUWB system with a single carrier system (SC) with fractionally spaced linear equalization at $500 \mathrm{MHz}$ and without equalization.

In Fig. 3, the BER performance of the OFDM-SUWB with channel coding and interleaving is examined over IEEE 802.15.4a CM3. BPSK modulation is used. Channel coding is performed through rate $1 / 2$ convolution codes with a corresponding hard Viterbi decoder at the receiver with the trace back lengths $T_{\text {blength }} 3$ and 15 . We select the constraint length 3. The minimum free distance of the code for the chosen constraint length is 5 . The coder is associated with a matrix interleaver with dimensions $16 \times 4$. A corresponding 


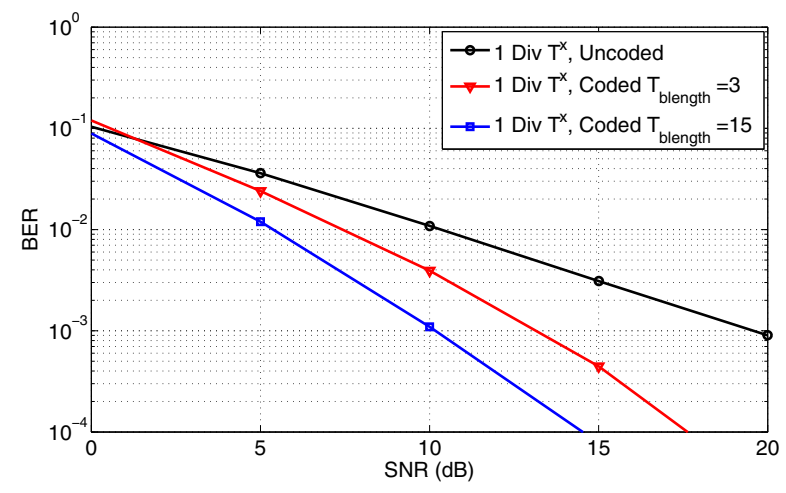

Fig. 3. BER vs. SNR performance of the OFDM based SUWB system with channel coding and interleaving.

de-interleaver is incorporated at the receiver. It is observed that with decoder $T_{\text {blength }}$ of 3 the SNR is improved by 7 $\mathrm{dB}$ at the BER of $10^{-3}$. With the free distance of 5 and code rate $1 / 2$, the expected coding gain is $3.89 \mathrm{~dB}$ [13]. The extra gain may be attributed to the interleaver. As discussed earlier, a major contributor for performance degradation is channel nulls. A good interleaver design randomizes the channel null errors such that the errors appear to be as independent errors and are within the correction capability of the codes.

In Fig. 4, the un-coded BER performance of the OFDMSUWB system is compared with that of the MB-OFDM system [2] over the IEEE 802.15.3a channel models CM1 and CM2 [8]. QPSK modulation is used. For a fair comparison the data rate in maintained the same i.e. around $53 \mathrm{Mbps}$. As can be seen from the results, the OFDM-SUWB system is able to achieve a comparable performance in addition to the significant power savings vis-a-vis MB-OFDM as described in Sec. III-C. For CM1, a BER of 0.01 is obtained at an SNR of around $8 \mathrm{~dB}$ for the OFDM-SUWB system whereas it is 6.5 $\mathrm{dB}$ in the case of the MB-OFDM system. It is also observed that the BER of the OFDM-SUWB system is better than the MB-OFDM system at lower SNRs with a cross over point at $5 \mathrm{~dB}$. For CM2, BER of 0.01 is obtained at an SNR of 9.5 $\mathrm{dB}$ for the OFDM-SUWB system whereas it is $6.8 \mathrm{~dB}$ in the case of the MB-OFDM system. In this channel the cross over point in the BER performance is at $4 \mathrm{~dB}$.

Moreover, it can be seen that the performance of the MBOFDM system is the same for both the channels. This is because MB-OFDM performs the complex FFT operation at 500 $\mathrm{MHz}$. The system is designed for high data rate operation with no scope to scale power with range/data rate to achieve desired performance especially at medium data rates. In contrast, the proposed OFDM-SUWB system shows differences depending on the channel delay spread. This is appealing as this adheres with the general notion of scalability of complexity with range/data rate to achieve desired performance [5].

\section{CONCLUSION}

In this work, a sub-sampled OFDM based SUWB system is proposed. The method is attractive as it enables mitigation of the fading channel at the sub-sampled rate. Moreover, it

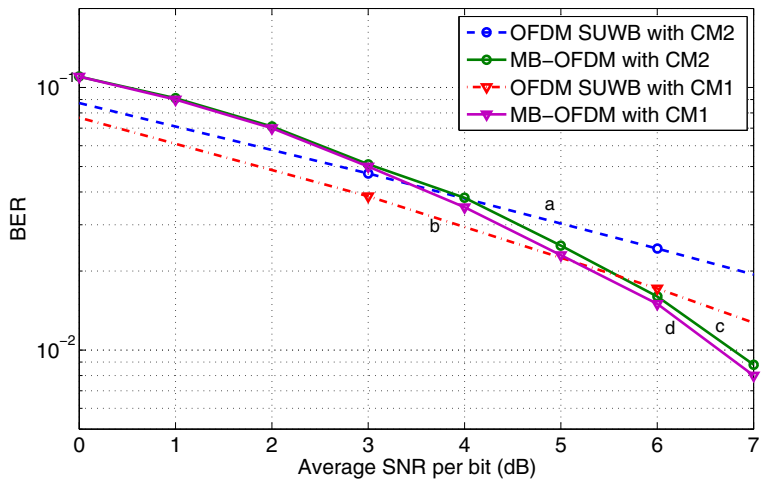

Fig. 4. BER vs. SNR performance comparison of the Sub-sampled OFDM SUWB system with the MB-OFDM system for the IEEE 802.15.3a CM1 and CM2 with rms delay spreads 5 ns and 8 ns respectively. (a) and (b) are for the OFDM SUWB system, (c) and (d) are for the MB-OFDM system

improves the flexibility in resource allocation as this can be done on a per subcarrier basis. Also it eases the stringent requirements on the code design when compared to the earlier SUWB system. The BER results indicate that the performance is promising especially at the lower SNRs. SNR improvements of the order of $2 \mathrm{~dB}$ and $8 \mathrm{~dB}$ were obtained for a BER of 0.01 when compared to the complex SUWB SC-LE system and SUWB system without equalization respectively. Also a BER performance comparable with the MB-OFDM system at $53 \mathrm{MBps}$ is obtained in addition to the significant reduction in power consumption due to reduced sampling rate requirements. Future work will focus on evolving the baseband algorithms and performance enhancement techniques.

\section{REFERENCES}

[1] M. Z. Win, and R. A. Scholtz, "impulse radio: how it works," IEEE Comm. Letters, vol. 2, no.2 February 2002.

[2] J. Balakrishnan et al, "Design of a multiband OFDM system for realistic UWB channel environments," IEEE Trans. on Microwave Theory and Techniques, vol. 52,no. 9, pp. 2123-2138, Sept 2004.

[3] K. Bynam, J.P. Nair D. Sen, R. Sinha and A. Naniyat, "A sub-band based technique for low power medium data rate ultra wide band communication," Proc. of IEEE VTC 2010-Spring,16-19 May 2010.

[4] D. Sen, J. P. Nair, K. Bynam, R. Sinha, and A. Naniyat, "An energy efficient sub-band based UWB receiver and its performance improvement by interference rejection filtering," Proc. of IEEE ICC Workshops 2010 , Cape Town, May 2010.

[5] J. Oh, Z. J. Haas, "Personal environment service based on the integration of mobile communications and wireless personal area networks," IEEE Comm. Magazine, pp. 66-73, June 2010.

[6] D. Cabric, M. S. W. Chen, D. A. Sobel, S. Wang, J. Yang, R. W. Brodersen, "Novel radio architectures for UWB, $60 \mathrm{GHz}$, and cognitive wireless systems," EURASIP Journal on Wireless Communications and Networking, vol. 2006, no.2, pp. 1-18, June 2006

[7] A.F Molisch et al, "IEEE 8021.5.4a channel model - final report"

[8] Jeffrey Foerster et al, "Channel modeling sub-committee report final," IEEE P802.15-02/490r1-SG3a02/368r5-SG3a, February 2003.

[9] S.W Goloumb and G. Gong, "Signal design with good correlation for wireless communications, cryptography and radar," Cambridge University Press, 2004.

[10] L. J. Cimini,"Analysis and simulation of a digital mobile channel using orthogonal frequency division multiplexing," IEEE Trans. on Communications, vol. 33, no. 7, pp. 665-675, July 1985.

[11] Bin Le, et. al, "Analog to digital converters," IEEE Signal Processing Magazine, pp. 69-77, Nov. 2005.

[12] S.M. Kay, "Fundamentals of statistical signal processing, Vol. I estimation theory," Prentice Hall, 1993.

[13] J. G. Proakis, "Digital communications," McGraw Hill, 2000. 